\title{
Towards In-Vivo Characterization and Deep Imaging of the Cornea and Beyond
}

\author{
Kristina Irsch \\ Institut de la Vision, Sorbonne University, Paris, France \\ Quinze-Vingts National Eye Hospital, Paris, France \\ The Wilmer Eye Institute, The Johns Hopkins University School of Medicine, Baltimore, Maryland \\ kristina.irsch@upmc.fr
}

\begin{abstract}
This talk reviews ongoing work towards in-vivo characterization and deep imaging of the human eye, primarily for the purpose of quantifying corneal transparency, for which to date no objective clinical tool exists. (C) 2018 The Author(s)

OCIS codes: (170.0170) Medical optics and biotechnology; (170.4460) Ophthalmic optics and devices; (170.4470) Ophthalmology; (170.4500) Optical coherence tomography; (330.4300) Vision system - noninvasive assessment; (330.7327) Visual optics, ophthalmic instrumentation.
\end{abstract}

\section{Introduction}

The diagnosis and management of ophthalmic diseases has been transformed in the last decade by the advent of optical coherence tomography (OCT) [1]. Ophthalmic OCT uses interference of broadband or tunable light to generate optical sections of the retina and anterior segment. However, conventional (time- or spectral-domain) clinical OCT systems generally suffer from limited lateral resolution and quantification capabilities.

This talk reviews ongoing work towards in-vivo characterization and deep imaging of the human eye, primarily based on full-field optical coherence tomographic microscopy (FF-OCT or FFOCM, a high-resolution variant of OCT) and for the purpose of quantifying corneal transparency, for which to date no objective clinical tool exists.

\section{Methods and Results}

Full-field optical coherence tomographic microscopy (FF-OCT or FF-OCM [2,3]), originally developed by Prof. Claude Boccara's group at the Institut Langevin, uses high numerical-aperture optics and acquires en face (parallel to the tissue surface) tomographic images without $x-y$ scanning, thereby achieving a lateral resolution comparable to the resolution in depth. A spinoff from Institut Langevin (LLTech, SAS) has commercialized FF-OCT for in-vitro microscopy.

At the Quinze-Vingts National Eye Hospital (in collaboration with Prof. Vincent Borderie's anterior segment service), we have been investigating the device's potential for examining donor corneas before transplantation, as an addition and improvement upon current eye-banking procedures for graft quality control [4]. In fact, we have already demonstrated the capability of FF-OCT to provide structural information superior to histology and to identify more disease indicators as was possible with the combination of clinical OCT and confocal microscopy [5]. Further, the technique was able to identify new quantitative parameters in the assessment of human donor corneal stroma $[4,5]$ (note that the transparency of the cornea largely relates directly to stromal structure [6,7]). Most notably, objective analysis of stromal light backscattering with FF-OCT (also in collaboration with Prof. Karsten Plamann from the Laboratoire d'Optique et Biosciences) has shown promise in the quantification of corneal transparency, as well as in differentiating abnormal from normal corneal tissues [8].

In collaboration with Prof. Boccara's group at the Institut Langevin, ongoing work [9] seeks to make FF-OCT suitable for in-vivo characterization of the cornea, with the development of a non-contact system that integrates an ultrahigh-speed CMOS camera. Contrary to confocal microscopy [10], axial resolution (determined by the light source bandwidth) and lateral resolution (determined by the resolving power of the objectives) are decoupled in FFOCT. Thus, FF-OCT can achieve non-contact operation with moderate numerical aperture objectives, and at the same time high lateral and axial resolutions down to $1 \mu \mathrm{m}$ along with a relatively large field-of-view.

To further enhance FF-OCT technology and enable deeper penetration into the corneal tissue and beyond, we are also exploring axial motion compensation (collaboration with Prof. Jin Kang, at the Johns Hopkins Whiting School of Engineering [11]) and light manipulation (collaboration with Dr. Alexandre Aubry, at the Institut Langevin $[12,13]$, an expert in light manipulation techniques, in particular the backscattering matrix approach $[14,15])$ techniques.

\section{Discussion and Conclusion}

These advances will establish the foundation for and the development of new clinical tools and diagnostic procedures permitting the objective and quantitative characterization of corneal transparency. Loss of corneal 
transparency is a major cause of visual handicap worldwide, affecting over 10 million people [16]. While early diagnosis and quantitative follow-up could improve clinical outcome and hence prevent loss of vision, means to assess corneal transparency are extremely limited and in current clinical and eye-bank practice usually involve a subjective and qualitative observation of opacities. Hence, the ability to objectively and quantitatively assess and monitor changes in corneal transparency will have a substantial impact in the study, prevention, diagnosis, treatment and monitoring of various pathologies as well as surgeries affecting corneal transparency.

Beyond the characterization of corneal transparency, the presented research will open the door to the characterization of transparencies of other ocular media (most notably of the crystalline lens, which will be important to understand the various aspects and factors in cataract development). In addition, the technological FFOCT enhancements, specifically the implementation of a backscattering matrix approach for eye imaging [12,13] that takes advantage of recent advances in light-manipulation and wavefront-shaping techniques [14,15], have implications beyond that of ocular media transparency characterization, most notably for imaging through ocular media opacities (e.g., imaging the retina when there is loss of ocular media transparency, such as imaging through cataract opacities).

\section{Acknowledgments}

The presented work is funded by the European Union's Horizon 2020 research and innovation programme under the Marie Skłodowska-Curie grant agreement No. 709104. In addition to the collaborators named in the paper, and the students and postdoctoral fellows who participated in this research (most notably Romain Bocheux, Viacheslav Mazlin, and Victor Barolle), the author gratefully acknowledges and thanks Profs. José-Alain Sahel and Mathias Fink, from the Institut de la Vision and Institut Langevin respectively, for their help with and support of the research and collaborations (which have emerged mainly due to the synergies created within the scope of their HELMHOLTZ project, funded by the European Research Council under the Synergy grant agreement No. 610110).

\section{References}

[1] J. Fujimoto and E. Swanson, "The development, commercialization, and impact of optical coherence tomography," Invest. Ophthalmol. Vis. Sci. 57, OCT1-OCT13 (2016).

[2] E. Beaurepaire, A. C. Boccara, M. Lebec, L. Blanchot, and H. Saint-Jalmes, "Full-field optical coherence microscopy,” Opt. Lett. 23, 244-246 (1998).

[3] A. Dubois, L. Vabre, A. C. Boccara, and E. Beaurepaire, "High-resolution full-field optical coherence tomography with a Linnik microscope." Appl. Opt. 41, 805-812 (2002).

[4] K. Irsch, K. Grieve, M. Borderie, D. Ghoubay, C. Georgeon, and V. Borderie, "Full-field optical coherence microscopy for histology-like analysis of stromal features in corneal grafts," J. Vis. Exp. (in press).

[5] M. Borderie, K. Grieve, K. Irsch, C. Georgeon, D. Ghoubay, C. Desousa, L. Laroche, and V. Borderie, "New parameters in assessment of human donor corneal stroma," Acta Ophthalmol. 95, e297-e306 (2017).

[6] D. Maurice, “The structure and transparency of the cornea,” J. Physiol. 136, 263-86 (1957).

[7] K. Plamann, F. Aptel, C. L. Arnold, A. Courjaud, C. Crotti, F. Deloison, F. Druon, P. Georges, M. Hanna, J. M. Legeais, F. Morin, É. Mottay, V. Nuzzo, D. A. Peyrot, and M. Savoldelli, "Ultrashort pulse laser surgery of the cornea and the sclera," J. Opt. 12, 084002 (2010).

[8] K. Irsch, M. Borderie, K. Grieve, K. Plamann, L. Laroche, and V. Borderie,. "Objective analysis of stromal light backscattering with full-field optical coherence tomographic microscopy shows potential to quantify corneal transparency," in Frontiers in Optics 2015, OSA Technical Digest (Optical Society of America, 2015), paper FW6A.6.

[9] V. Mazlin, P. Xiao, E. Dalimier, K. Grieve, K. Irsch, J. A. Sahel, M. Fink, and A. C. Boccara, "In vivo high resolution human corneal imaging using full-field optical coherence tomography,” Biomed. Opt. Express 10, 557-568 (2018).

[10] J. Stave, G. Zinser, G. Grummer, and R. Guthoff, "Modified Heidelberg retinal tomograph HRT. Initial results of in vivo presentation of corneal structures," Ophthalmologe 99, 276-280 (2002).

[11] K. Irsch, S. Lee, S. N. Bose, and J. U. Kang, "Motion-compensated optical coherence tomography using envelope-based surface detection and Kalman-based prediction," Proc. SPIE 1084, 104840Q (2018).

[12] V. Barolle, A. Badon, C. Boccara, M. Fink, A. Aubry, and K. Irsch, "Matrix Approach of Eye Optical Imaging," in Imaging and Applied Optics 2017, OSA Technical Digest (Optical Society of America, 2017), paper MW3C.2.

[13] V. Barolle, A. Badon, L. Cobus, K. Irsch, C. Boccara, M. Fink, and A. Aubry, "Matrix approach of optical coherence tomography for indepth imaging of biological tissues," in Imaging and Applied Optics 2018, OSA Technical Digest (Optical Society of America, 2018), paper JTu5B.7.

[14] A. Aubry and A. Derode "Detection and imaging in a random medium: A matrix method to overcome multiple scattering and aberration," J. Appl. Phys. 106, 044903 (2009).

[15] A. Badon, D. Li, G. Lerosey, A. C. Boccara, M. Fink, and A. Aubry "Smart optical coherence tomography for ultra-deep imaging through highly scattering media," Sci. Adv. 2, e1600370 (2016).

[16] J. P. Whitcher, M. Srinivasan, and M. P. Upadhyay, “Corneal blindness: a global perspective,” Bull. World Health Organ. 79, 214-221 (2001). 